\title{
Combining light polarization and speckle measurements with multivariate analysis to predict bulk optical properties of turbid media
}

\author{
DAPHNÉ HÉRAN ${ }^{1, *}$, MAXIME RYCKEWAERT ${ }^{1}$, YANNICK ABAUTRET ${ }^{2}$, MYRIAM \\ ZERRAD $^{2}$, Claude AMRA ${ }^{2}$ AND Ryad BENDOULA ${ }^{1}$ \\ ${ }^{1}$ ITAP, IRSTEA, Montpellier SupAgro, Université Montpellier, France \\ ${ }^{2}$ Aix Marseille Univ, CNRS, Centrale Marseille, Institut Fresnel, 13013 Marseille, France \\ *daphne.heran@irstea.fr
}

\begin{abstract}
This study aims to investigate the combination of speckle pattern analysis, polarization parameters and chemometric tools to predict the optical absorption and scattering properties of materials. For this purpose, an optical setup based on light polarization and speckle measurements was developed and turbid samples were measured at $405 \mathrm{~nm}$ and 660 $\mathrm{nm}$. First, backscattered polarized speckle acquisition was performed on a set of 41 samples with various scattering $\left(\mu_{s}\right)$ and absorbing $\left(\mu_{a}\right)$ coefficients. Then, several parameters were computed from the polarized speckle images and prediction models were built using stepwise-Multiple Linear Regression. For scattering media, $\mu_{s}$ was predicted with $\mathrm{R}^{2}>0.9$ using two parameters. In the case of scattering and absorbing media, prediction results using two parameters were $\mathrm{R}^{2}=0.62$ for $\mu_{s}$ and $\mathrm{R}^{2}=0.8$ for $\mu_{a}$. The overall results obtained in this research showed that the combination of speckle pattern analysis, polarization parameters and chemometric tools to predict the optical bulk properties of materials show interesting promises.
\end{abstract}

OCIS codes: (030.6140) Speckle; (290.5850) Scattering, particle; (260.5430) Polarization; (170.1580) Chemometrics; (160.4760) Optical properties; (170.7050) Turbid media

C 2018 Optical Society of America

\section{Introduction}

Non-invasive determination of optical absorption and scattering properties of materials is a subject of considerable interest for biomedical $[1,2]$, agricultural $[3,4]$ or chemical $[5,6]$ applications. When these two optical properties are obtained separately, the individual roles played by absorption and scattering in light matter interactions are better understood. Moreover, absorption and scattering coefficient knowledge can be useful for qualitative and quantitative analysis.

Specific experimental techniques based on light propagation theory and radiative transfer equation resolution have been proposed to measure absorption and scattering properties separately. These techniques involved different setups based on spatially-resolved [7] timeresolved $[8,9]$ and frequency-resolved $[10]$ spectroscopy as well as double integrating sphere setups $[11,12]$ or polarization metrology $[13,14]$.

Although powerful, these methods have their limitations. First, they may require complex and sometimes expensive optical solutions. Secondly, the model inversion technique used to obtain the absorption and scattering coefficients requires the knowledge or approximation of parameters describing the studied medium (such as thickness, refractive index, particle size and shape, etc.), which may be troublesome for complex media $[11,15]$.

Laser speckle theory was first developed in the 1960s [16]. Statistical properties of laser speckle pattern are related to the scattering characteristics of an optically diffuse material. Speckle field measurement is now a standard tool used to characterize dynamic behavior of scattering media. Indeed, well-known techniques such as Dynamic Light Scattering [17], 
Diffusing Wave Spectroscopy [18], LAser Speckle Contrast Analysis (LASCA) [19] based on temporal characterization of speckle intensity fluctuations are now widely used for diverse applications such as medicine [20] or agriculture [21].

Besides, speckle pattern parameters (typical size, contrast, intensity...) combined with a polarization approach can provide additional information on scattering media [22-28]. For example, spatial intensity variations, speckle size, image spatial contrast or degree of polarization of speckle patterns were shown to be dependent on the anisotropy factor and scattering coefficient of the studied medium [29, 30]. More recently, speckle pattern parameters combined with a polarization approach [31,32] has been carried out on both scattering and absorbing media. It showed that the speckle pattern parameters of a scattering medium were also dependent on the absorption coefficient. These first results are very encouraging. Measuring speckle pattern parameters can indeed potentially reveal the absorption and scattering properties of materials.

In analytical chemistry, relationships between multivariate data and media properties are commonly described by using chemometrics methods. To our knowledge, the combination of speckle pattern analysis, polarization parameters and chemometric tools to predict the optical absorption and scattering properties of materials has never been tested. In this study, the potential of this approach is evaluated on turbid media. This paper addressed the following issues: (i) select relevant speckle pattern parameters to (ii) build and evaluate prediction models based on these parameters. The results of this study are first presented on onlyscattering media and then on both scattering and absorbing media.

\section{Materials and methods}

\subsection{Optical phantoms}

\subsubsection{Liquid optical phantoms preparation}

A set of 41 liquid optical phantoms (Fig. 1) was prepared based on the protocol described in [33]. Absorbing properties were set by Methylene Blue (M9140, batch MKBR892V, SigmaAldrich), MB, and scattering properties set by IntraLipid ${ }^{\circledR} 20 \%$ (batch 10IB7209, Fresenius Kabi), IL. All solutions were diluted in deionized water to obtain $100 \mathrm{ml}$ samples.

A MB stock solution of $400 \mu \mathrm{M}$ was used to prepare seventeen absorbing levels with respectively $0,1,2.2,4,5,8,10,12,15,16,18,20,25,28,30,32$ and $36 \mathrm{ml}$ of stock solution. The corresponding MB concentrations were then: $0,4,8.8,16,20,32,40,48,60$, $64,72,80,100,112,120,128$ and $144 \mu \mathrm{M}$.

Similarly, ten scattering levels were created with $1,2,3,4,5,6,7,8,9$ and $10 \mathrm{ml}$ of IL $20 \%$. IL $20 \%$ is originally developed for intravenous feeding and contains soybean oil (200 $\mathrm{g} /$ liter $)$, emulsified with egg lecithin (12 g/liter) in water-glycerin mixture. Taking into account the density of the different components, this results in a volume concentration of $22.7 \%$ scattering particles in pure IL $20 \%[33,34]$. As a consequence, scattering particle concentrations in the samples were $0.227,0.454,0.681,0.908,1.135,1.362,1.589,1.816$, 2.043 and $2.270 \%$ (volume fraction). Batch-to-batch variability in IL scattering properties has been proven to be very small and its high stability over time and at different temperatures makes it suitable for calibration of setups developed for optical characterization [35-37]. 


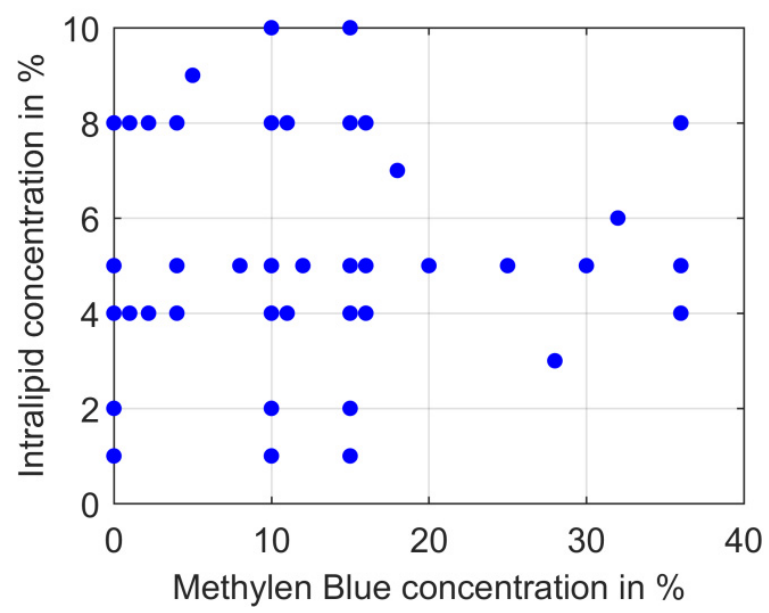

Fig. 1. Set of 41 liquid phantoms, created by mixing Intralipid® $20 \%$ (IL; scatter), Methylene Blue (MB; absorber) and water (dilution agent).

\subsubsection{Estimation of bulk optical properties}

Scattering coefficients $\mu_{s}$ at $660 \mathrm{~nm}$ and $405 \mathrm{~nm}$ for pure Intralipid $₫ 20 \%$ were computed according to the fitted equation given in [33]:

$$
\mu_{s}(\lambda)=1.868 \times 10^{10} \times \lambda^{-2.59}
$$

with $\mu_{s}$ in $\mathrm{cm}^{-1}$ and $\lambda$ in $\mathrm{nm}$.

This equation was established with optical measurements on similar samples between 500 $\mathrm{nm}$ and $2500 \mathrm{~nm}$. It was then extrapolated to $405 \mathrm{~nm}$ as other studies such as [34] reported similar $\mu_{s}$ model between $350 \mathrm{~nm}$ and $500 \mathrm{~nm}$.

The scattering coefficients for the different IL concentrations were then computed using the linear relation between scattering and volume concentration for the scattering particles. This linear relation is valid for solutions with less than $2 \%$ of scattering particles especially for wavelengths in the near infrared. Two of the liquid samples prepared were slightly above this limit but as measurements were done in the visible range, the impact was considered negligible.

Absorption coefficients $\mu_{a}$ at $665 \mathrm{~nm}$ of solutions were also computed thanks to the fitted linear equation found in [33].

$$
\mu_{a}=0.1071 \times C_{M B}
$$

with $C_{M B}$ the Methylene Blue concentration.

This linear fit is valid for MB concentrations lower than $70 \mu \mathrm{M}$. Above this limit, the fraction of absorbers around $665 \mathrm{~nm}$ (monomers) decreases significantly in favor of the $\mathrm{MB}$ dimers fraction [38].

In this experiment, absorption coefficients of solutions with MB concentrations higher than $70 \mu \mathrm{M}$ were estimated by a linear fit between the value at $70 \mu \mathrm{M}$ and the value at 144 $\mu \mathrm{M}$ found in the article [33]. At $405 \mathrm{~nm}, \mathrm{MB}$ absorption is negligible [39].

\subsection{Experimental setup and parameter computations}

\subsubsection{Experimental setup}

The optical setup used for backscattered speckle measurements is schematically illustrated in Fig. 2. 


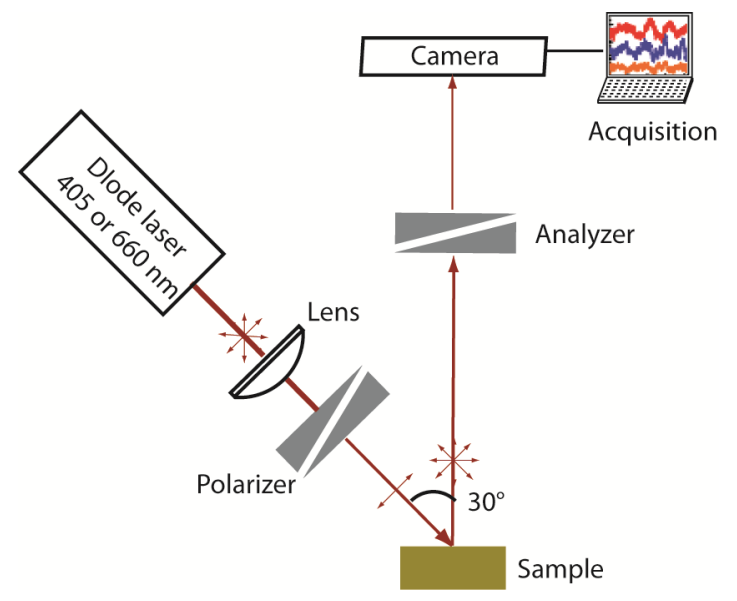

Fig. 2. Experimental setup scheme of speckle measurements

Two laser diodes were used in order to stimulate the liquid samples in two different wavelength ranges: one at $405 \mathrm{~nm}(40 \mathrm{~mW}$, Thorlabs DL5146-101S) where absorption was negligible and one at $660 \mathrm{~nm}(120 \mathrm{~mW}$, Thorlabs HL6545MG) where both absorption and scattering occurred. Both laser diodes were mounted with an aspheric lens (Thorlabs A220TM for the $405 \mathrm{~nm}$ laser diode and Thorlabs C220TME-B for the $660 \mathrm{~nm}$ laser diode) adjusted to minimize the laser spot at the sample surface .

A grid polarizer (Thorlabs WP12L-UB) was mounted between the laser diode and the sample to set a p-polarization and an analyzer (Thorlabs WP25M-UB) mounted in front of the camera (CMOS, Thorlabs DCC3240M) to measure backscattered speckle in p- and spolarization alternatively. The CMOS camera recorded the speckle field on $1024 \times 1280$ pixels of $5.3 \mu \mathrm{m} \times 5.3 \mu \mathrm{m}$ pitch. The distance between the sample and the camera was set to $20 \mathrm{~cm}$ so a typical speckle spot covered a few pixels. The image measured with parallel polarizer and analyzer was named $-p p$ and the image measured with crossed polarizer and analyzer was named $-p s$.

The integration time of the camera was set to $0.1 \mathrm{~ms}$ [40] and the frame rate was set to 60 fps to avoid blur on the image due to particle Brownian motion in the liquid sample. For each measurement, 100 frames were taken in a row.

A dark measurement was also performed for each liquid sample to remove the background signal on the images.

\subsubsection{Speckle pattern and polarization parameters}

Several statistical parameters related to the speckle pattern for each polarization can be extracted from the speckle images. These parameters were computed for the 100 frames and then averaged on the number of realizations to give the results for one measurement.

First of all, the average intensity of the images $-p p$ and $-p s$ were computed: $\left\langle I_{p p}\right\rangle,\left\langle I_{p s}\right\rangle$. $<I_{p p}>$ is the sum of the polarization maintaining light and half of the depolarized light, whereas $\left\langle I_{p s}\right\rangle$ corresponds to half of the depolarized light [41]. The average surface and volume intensity, respectively $I_{\text {surf }}$ and $I_{v o l}$ were then defined as:

$$
\begin{gathered}
I_{s u r f}=<I_{p p}>-<I_{p s}> \\
I_{v o l}=2 .<I_{p s}>
\end{gathered}
$$

The degree of linear polarization $\left(D O P_{l}\right)$, which gives information about the predominant type of photons, can also be computed to characterize samples. It was given by:

$$
D O P_{l}=\frac{<I_{p p}>-<I_{p s}>}{<I_{p p}>+<I_{p s}>}
$$


Besides, in [16, 42] the "average width" of a speckle pattern is determined from calculations of the normalized autocorrelation function of the intensity distribution in the $(\mathrm{x}, \mathrm{y})$ plane. This function, denoted $c_{I}(\Delta x, \Delta y)$ was calculated from the intensity distribution of the measured speckle, $I(x, y)$ :

$$
c_{I}(\Delta x, \Delta y)=\frac{F T^{-1}\left[|F T[I(x, y)]|^{2}\right]-\langle I(x, y)\rangle^{2}}{\left\langle I(x, y)^{2}\right\rangle-\langle I(x, y)\rangle^{2}}
$$

where $F T$ was the Fourier Transform, $<$. $>$ was a spatial average.

We define $c_{I}(\Delta x, 0)$ and $c_{I}(0, \Delta y)$ the horizontal and vertical profiles of $c_{I}(\Delta x, \Delta y)$, respectively. The full width at half maximum of this function provides a reasonable measure of the speckle size. Given the setup geometry, only the width along the vertical profile ( $d y$ such as $\left.c_{I}(0, d y / 2)=0.5\right)$ was considered, to avoid the incident angle influence. For each sample, two speckle sizes were computed in micrometers, depending on the analyzed polarization state: $d y_{p p}$ and $d y_{p s}$. Moreover, the difference $d y_{p p}-d y_{p s}$ has been investigated in the case of volume scattering and absorbing media characterization [31].

One last parameter extracted from speckle images $-p p$ or $-p s$ was the contrast $\left(C_{p p}\right.$ or $\left.C_{p s}\right)$ defined as [16]:

$$
C=\frac{\sigma_{I}}{\langle I(x, y)\rangle}
$$

where $\sigma_{I}$ was the square root of the intensity variance and $<$. $>$ was a spatial average.

\subsection{Multivariate Analysis}

Stepwise Multiple Linear Regression (Stepwise-MLR) is commonly used for variable selection [50]. This procedure retains a subset of the most relevant observed variables to establish a regression model. The response variable is then predicted from a linear combination of the variables selected. The linear regression model can be written as:

$$
y_{j}=\beta_{0}+\sum_{i=1}^{N} \beta_{i} x_{i}+\varepsilon_{j}
$$

Where $y_{j}$ is a response variable, $x_{i}$ a dataset of $\mathrm{N}$ observed variables, $\beta_{k}(\mathrm{k}=0, \mathrm{~N})$ the regression coefficients and $\epsilon_{j}$ the residuals.

In this study, the stepwise-MLR was applied to fit scattering and absorption coefficients from speckle parameters. More precisely, variable selection was performed on eight potential predictor parameters $\left(d y_{p p}, d y_{p s}, d y_{p p}-d y_{p s}, C_{p p}, C_{p s}, D O P, I_{s u r f}, I_{v o l}\right)$ to predict bulk optical properties.

Three quarters of the samples ( 30 samples) were used to build the calibration models and define the model parameters. The second independent dataset, composed by one quarter of the samples (11 samples), was used to test the ability of the regression models to predict either scattering or absorption coefficients of this new dataset. Model performances were evaluated on the basis of coefficient of determination $\left(\mathrm{R}^{2}\right)$ and root mean square error of calibration or prediction (RMSEc or RMSEp).

All computations and multivariate data analysis were performed with Matlab software v.R2015b (The Mathworks Inc., Natick, MA, USA).

\section{Results and discussion}

\subsection{Optical sample properties}

From Eq. (1) and Eq. (2), the optical properties of the sample set are calculated and their statistics are presented in Table 1. 
Table 1: Average, dispersion and range of bulk optical properties obtained for each wavelength

\begin{tabular}{|c|c|c|c|c|c|}
\hline Wavelength & Coeff. & $\begin{array}{c}\text { Mean in } \\
\mathrm{cm}^{-1}\end{array}$ & $\begin{array}{c}\text { Std in } \\
\mathrm{cm}^{-1}\end{array}$ & $\begin{array}{c}\text { Min in } \\
\mathrm{cm}^{-1}\end{array}$ & $\begin{array}{c}\text { Max in } \\
\mathrm{cm}^{-1}\end{array}$ \\
\hline \multirow{2}{*}{$405 \mathrm{~nm}$} & $\mu_{s}$ & 174.47 & 80.15 & 33 & 329.7 \\
\hline \multirow{2}{*}{$660 \mathrm{~nm}$} & $\mu_{s}$ & 49.23 & 22.62 & 9.3 & 93.1 \\
\cline { 2 - 6 } & $\mu_{a}$ & 5.16 & 3.94 & 0 & 13 \\
\hline
\end{tabular}

At $405 \mathrm{~nm}$, the scattering behavior of samples is studied over a wide range of $\mu_{s}$ values: from $33 \mathrm{~cm}^{-1}$ to $329.7 \mathrm{~cm}^{-1}$, with a mean value of $174.47 \mathrm{~cm}^{-1}$. At $660 \mathrm{~nm}, \mu_{s}$ values range from $9.3 \mathrm{~cm}^{-1}$ to $93.1 \mathrm{~cm}^{-1}$ with a mean value of $49.23 \mathrm{~cm}^{-1}$ and $\mu_{a}$ values range from $0 \mathrm{~cm}^{-1}$ to $13 \mathrm{~cm}^{-1}$ with a mean value of $5.16 \mathrm{~cm}^{-1}$. Samples at $660 \mathrm{~nm}$ exhibit both scattering and absorbing behaviors, but $\mu_{s}$ values are not as high as $405 \mathrm{~nm}$ ones. Indeed IL is composed of spherical particles with typical size lower than $500 \mathrm{~nm}$ [33], which explains why $\mu_{s}$ at $405 \mathrm{~nm}$ is higher than $\mu_{s}$ at $660 \mathrm{~nm}$ for a given IL concentration.

It should be noted that in this experiment, $\mu_{s}$ coefficient is linear with IL concentration. Indeed, scattering level variations are only due to IL particle concentrations and not to particle size variations.

If the measured media were composed of particles of different sizes, it would have been preferable to work with the reduced scattering coefficient $\mu_{s}^{\prime}$ which takes into account the medium anisotropy factor $g\left(\mu_{s}^{\prime}=\mu_{s}(1-g)\right)[40]$ and to study other types of polarization, such as circular polarization [31].

\subsection{Speckle parameter selection and calibration models}

\subsubsection{Case of scattering media}




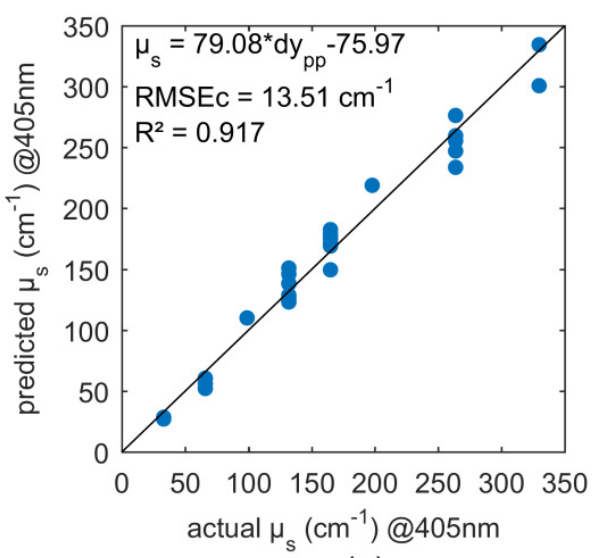

(a)

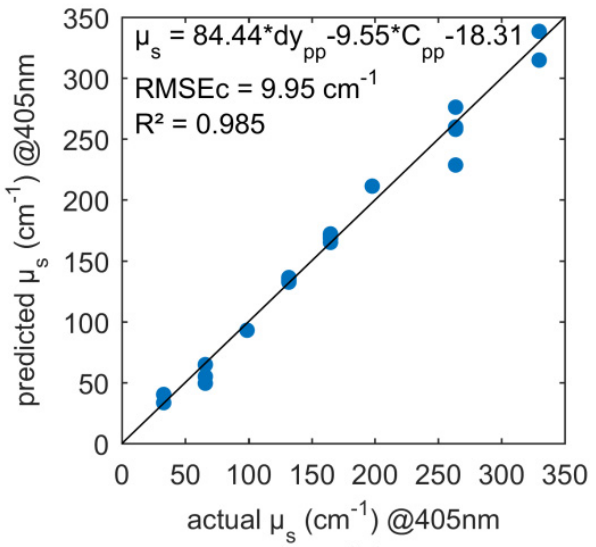

(b)

Fig. 3. Stepwise MLR predicted values vs actual values of $\mu_{s}$ at $405 \mathrm{~nm}$ using one (Fig. 3.a) and two (Fig. 3.b) parameters. The actual values are computed with Eq. (1)

At $405 \mathrm{~nm}$, the calibration equations obtained by the stepwise-MLR using one and two parameters are respectively:

$$
\begin{gathered}
\mu_{s}(405 \mathrm{~nm})=79.08 \times d y_{p p}-75.97 \\
\mu_{s}(405 \mathrm{~nm})=84.44 \times d y_{p p}-9.55 \times C_{p p}-18.31
\end{gathered}
$$

At this wavelength, where absorption is negligible compared to scattering, we find that $\mu_{s}$ is mainly linked to the parameter $d y_{p p}$ [Eq.(9)]. More precisely, $d y_{p p}$ increases with $\mu_{s}$.

This has already been seen in [40] in the case of non-polarized light. Indeed, the speckle spot size increases when the reflected light area from which it originates decreases:

$$
d y=\frac{1.22 \times \lambda \times D}{D_{e}}
$$

where $D$ is the observation distance and $D_{e}$ the light diffusing area diameter [43].

Moreover, in the multiple scattering regime, when light penetrates a sample, it propagates up to a depth of a few transport mean paths $l^{*}$ (where $l^{*}=1 /\left(\mu_{s}(1-g)\right)$ and $g$ is the anisotropy factor of the medium) and spreads out of the sample over an area $S_{e}$ of roughly $10 \times l^{* 2}$ [44, 45]. Consequently: 


$$
\begin{gathered}
S_{e} \approx \frac{10}{\left(\mu_{s}(1-g)\right)^{2}} \\
\pi \frac{D_{e}^{2}}{4} \approx \frac{10}{\left(\mu_{s}(1-g)\right)^{2}}
\end{gathered}
$$
$d y$.

By combining Eq. (11) and Eq. (13) we can establish a linear relationship between $\mu_{s}$ and

In this experiment, speckle patterns for two perpendicular polarizations of detection, $p p$ and $p s$ and the corresponding speckle sizes, $d y_{p p}$ and $d y_{p s}$ were measured. As stated by Morgan and Ridgway (Fig. 4), when linearly polarized light hits a medium, the backscattered light is composed of photons keeping the incident polarization state, and photons with random polarization states [46]. The photons having the incident polarization state have undergone a smaller number of scattering events and come from a smaller volume close to the surface compared to photons with a random polarization.
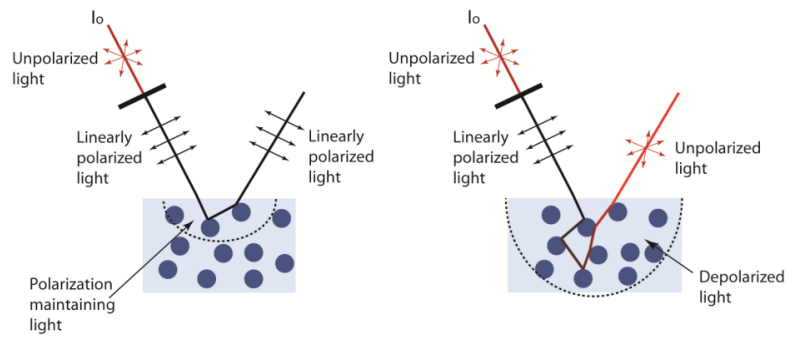

Fig. 4. Polarization state of light in a medium with linearly polarized incident light $[46,47]$.

As we can see in Fig. 5, most of the reflected light at $405 \mathrm{~nm}$ is depolarized $\left(D O P_{l}<0.1\right)$ and thus comes from the sample volume. As a consequence, $d y_{p p}$ and $d y_{p s}$ are both measurements of the depolarized speckle spot size. As we can see in Fig. 6, these variables provide the same information and are therefore redundant. The model [Eq.(9)] could use $d y_{p s}$ instead of $d y_{p p}$. But keeping both will not improve the predictive qualities.

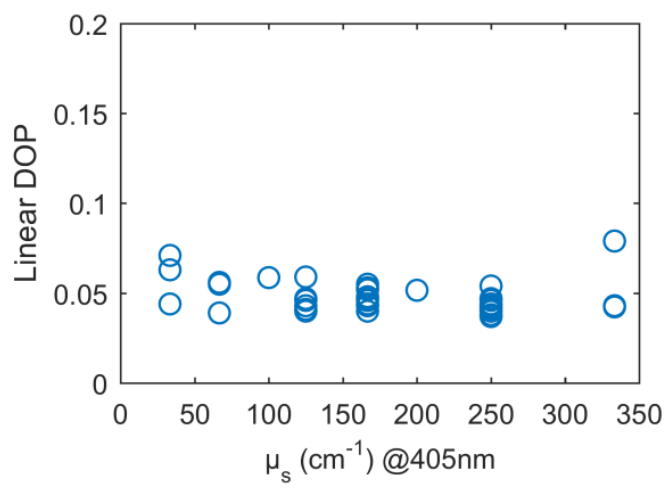

Fig. 5. Degree of linear polarization measured at $405 \mathrm{~nm}$ versus $\mu_{s}$ values. 


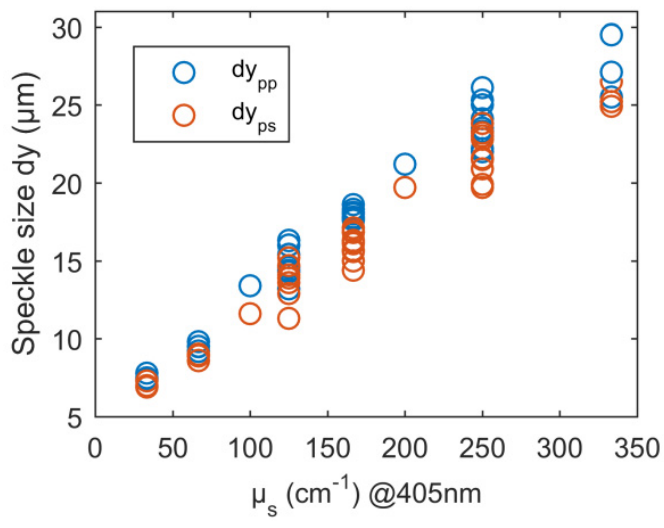

Fig. 6. Measured $d y_{p p}$ and $d y_{p s}$ versus $\mu_{s}$ values.

Furthermore, from the stepwise-MLR model, we can see that $\mu_{s}$ prediction is slightly improved $\left(\mathrm{R}^{2}\right.$ goes from 0.917 to $0.985, \mathrm{RMSE}_{\mathrm{c}}$ from 13.51 to $\left.9.95 \mathrm{~cm}^{-1}\right)$ when two parameters are taken into account: $d y_{p p}$ and $C_{p p}$. From the model equation, when the scattering level $\mu_{s}$ increases, the contrast $C_{p p}$ decreases [Eq. (10)].

According to [16], a simplified approach of the speckle contrast gives the following relationship:

$$
C=\frac{1}{\sqrt{N}}
$$

Where $C$ is the speckle pattern contrast and $N$ the number of independent speckles contributing to the speckle pattern (for example orthogonal polarized speckles or different wavelength contributions).

Previous works such as $[48,49]$ stated that scattering coefficients inside a volume can exhibit spectral variations. In our experiment, the spectral bandwidth of the laser diodes is about $2 \mathrm{~nm}$. Hence, given the source bandwidth, spectral variations of the sample scattering properties can lead to an independent speckle generation. Actually, it could be assumed that the more the scattering level, the more the number of independent speckles and therefore the lower the contrast.

\subsubsection{Case of scattering and absorbing media}

Scattering coefficient $\mu_{s}$ : speckle and polarization parameter selection and model calibration 


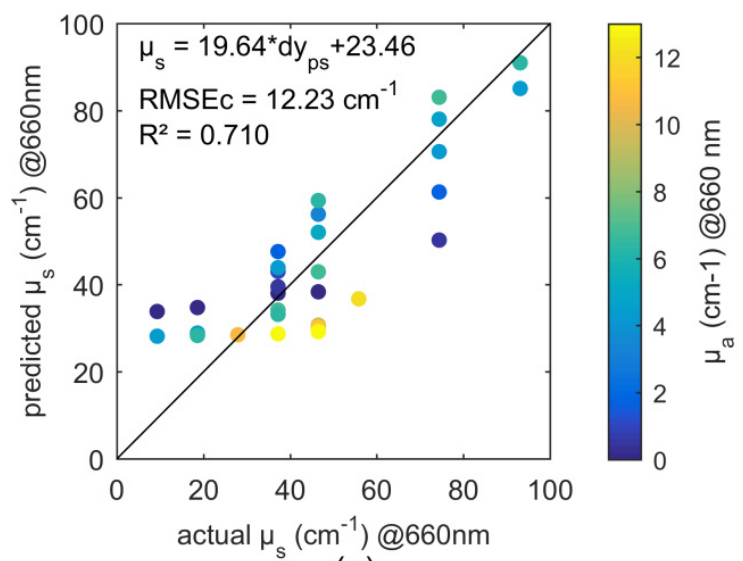

(a)

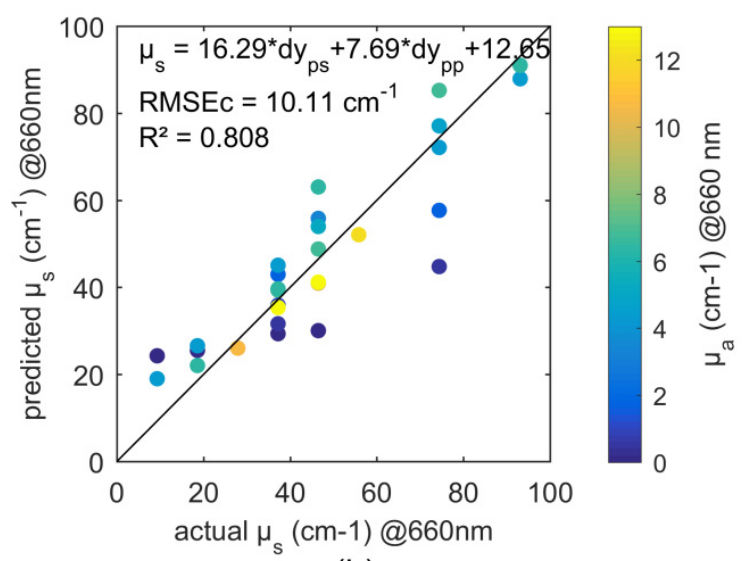

(b)

Fig. 7. Stepwise MLR predicted values vs actual values of $\mu_{s}$ at $660 \mathrm{~nm}$ using one (Fig. 7.a) and two (Fig. 7.b) parameters. The colorbar gives the level of absorption, $\mu_{a}$, for each point.

At $660 \mathrm{~nm}$, the $\mu_{s}$ calibration equations obtained by the stepwise-MLR using one and two parameters are respectively:

$$
\begin{gathered}
\mu_{s}(660 \mathrm{~nm})=19.64 \times d y_{p s}+23.46 \\
\mu_{s}(660 \mathrm{~nm})=16.29 \times d y_{p s}+7.69 \times d y_{p p}+12.65
\end{gathered}
$$

At this wavelength, all the samples both scatter and absorb. According to the stepwiseMLR model with one parameter, $\mu_{s}$ can be described with $d y_{p s}$ [Eq.(15)].

At first-order, the same analysis done previously linking $\mu_{s}$ at $405 \mathrm{~nm}$ to $d y$ can be done for $\mu_{s}$ at $660 \mathrm{~nm}$. However, here at $660 \mathrm{~nm}$, only $d y_{p s}$ gives a measurement of the depolarized speckle spot size. Indeed, $D O P_{l}$ at $660 \mathrm{~nm}$ range from 0.05 to 0.5 (Fig. 8.) while $D O P_{l}$ at 405 $\mathrm{nm}$ was around 0.05 for all samples (Fig. 5). This implies that a fraction of the light measured in $-p p$ configuration (polarizer and analyzer parallel) at $660 \mathrm{~nm}$ comes from the sample surface, where light has kept its incident polarization state (Fig. 4). This was not the case for $p p$ measurements done at $405 \mathrm{~nm}$. 


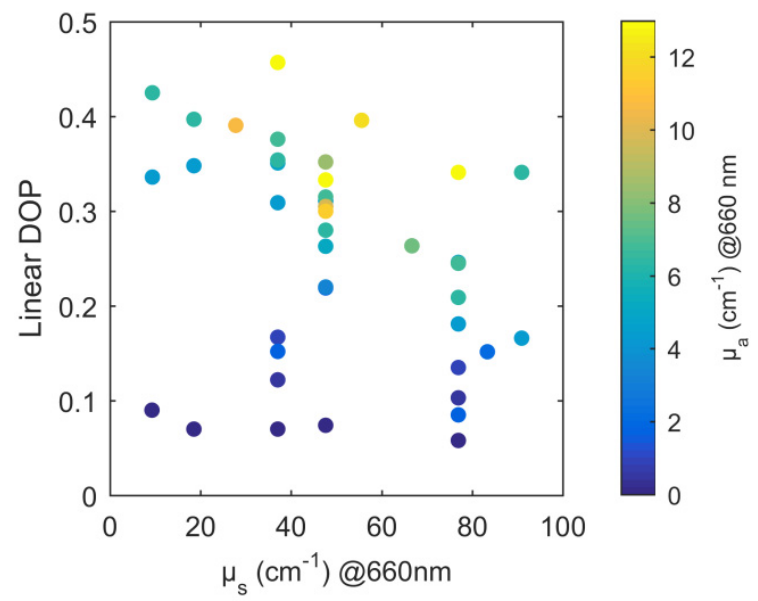

Fig. 8. Degrees of linear polarization measured at $660 \mathrm{~nm}$ versus $\mu_{s}$ values. The colorbar gives the level of absorption, $\mu_{a}$, for each point.

Moreover, as shown on Fig. 7, predicted values of $\mu_{s}$ at $660 \mathrm{~nm}$ can be greatly improved by using stepwise-MLR model with two parameters $\left(\mathrm{R}^{2}\right.$ goes from 0.710 to 0.808 , RMSEc from 12.23 to $10.11 \mathrm{~cm}^{-1}$ ): $d y_{p s}$ and $d y_{p p}$. These two parameters increase when $\mu_{s}$ at $660 \mathrm{~nm}$ increases. Adding $d y_{p p}$ parameter seems to improve $\mu_{s}$ prediction for high absorbing levels (yellow dots on Fig. 7). Indeed, when the absorption level is high, light is rapidly absorbed in the sample volume. Hence, the available information comes from the sample surface, characterized by $-p p$ measurements.

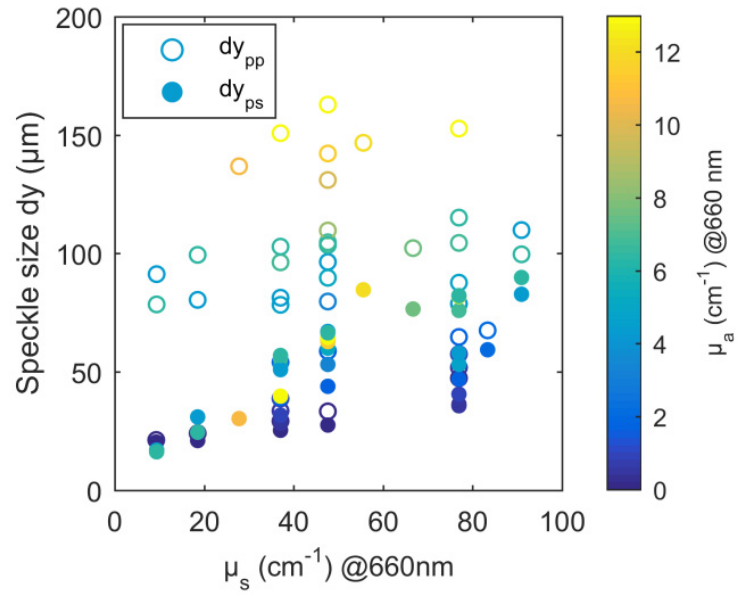

Fig. 9. Measured $d y_{p p}$ and $d y_{p s}$ versus $\mu_{s}$ values. The colorbar gives the level of absorption, $\mu_{a}$, for each point.

The $\mu_{s}$ model performances at $660 \mathrm{~nm}\left(\mathrm{R}^{2}=0.808\right)$ are not as good as those obtained at $405 \mathrm{~nm}\left(\mathrm{R}^{2}=0.985\right)$. Indeed, the sample absorption properties modify the penetration depth as well as the spread out area due to the scattering properties proportional to $d y$ value [Eq. (11) - Eq.(13)]. This can be seen in Fig. 9, where different samples with same $\mu_{s}$ and different $\mu_{a}$ give different $d y$ values.

Absorbing coefficient $\mu_{a}$ : speckle and polarization parameter selection and model calibration 


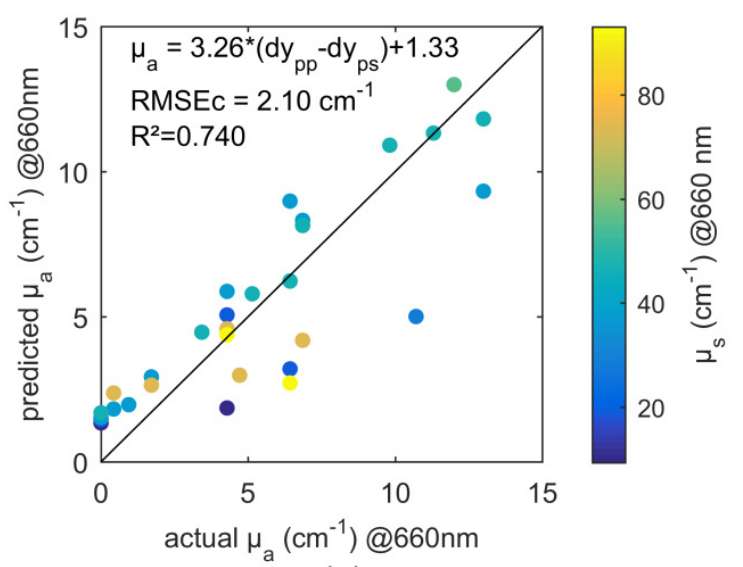

(a)

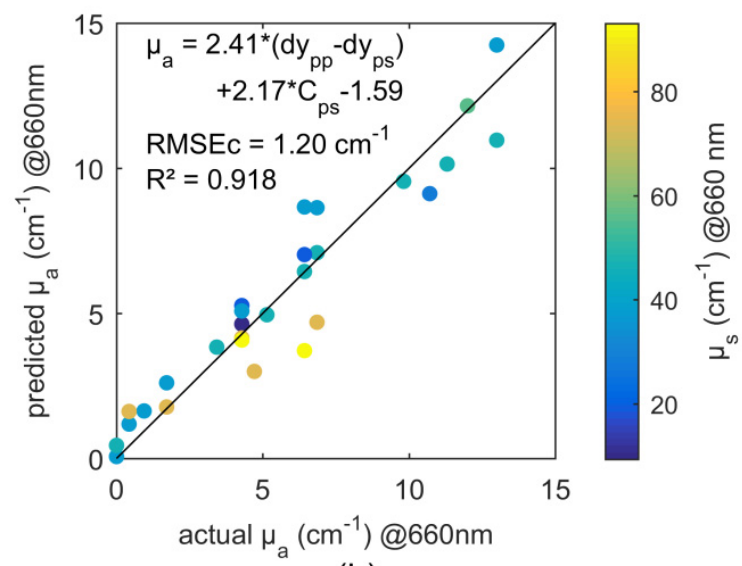

(b)

Fig. 10. Stepwise MLR predicted values versus actual values of $\mu_{a}$ at $660 \mathrm{~nm}$ using one (Fig. 10.a) and two (Fig. 10.b) parameters. The colorbar gives the level of scattering, $\mu_{s}$, for each point.

At $660 \mathrm{~nm}$, the $\mu_{a}$ calibration equations obtained by the stepwise-MLR using one and two parameters are respectively:

$$
\begin{gathered}
\mu_{\mathrm{a}}(660 \mathrm{~nm})=3.26 \times\left(\mathrm{dy}_{\mathrm{pp}}-\mathrm{dy}_{\mathrm{ps}}\right)+1.33 \\
\mu_{\mathrm{a}}(660 \mathrm{~nm})=2.41 \times\left(\mathrm{dy}_{\mathrm{pp}}-\mathrm{dy}_{\mathrm{ps}}\right)+2.17 \times \mathrm{C}_{\mathrm{PP}}-1.59
\end{gathered}
$$

According to the stepwise-MLR model with one parameter, $\mu_{a}$ can be described as a linear function of $d y_{p p}-d y_{p s}$. The linear increase tendency of $d y_{p p}-d y_{p s}$ with $\mu_{a}$ is well observed in Fig. 11. This kind of results was presented in [31] with four samples made of small particles (anisotropy factor $\mathrm{g}<0.3$ ). In this experiment, the IL anisotropy factor at $660 \mathrm{~nm}$ is equal 0.7 meaning that particles are not considered as small compared to the wavelength. However, the same tendency is observed.

The increase in $D O P_{l}$ with $\mu_{a}$ has also been shown in [31]. This implies that $\mu_{a}$ plays an important role in the depolarization process and on the size of the diffusion spread out area. 


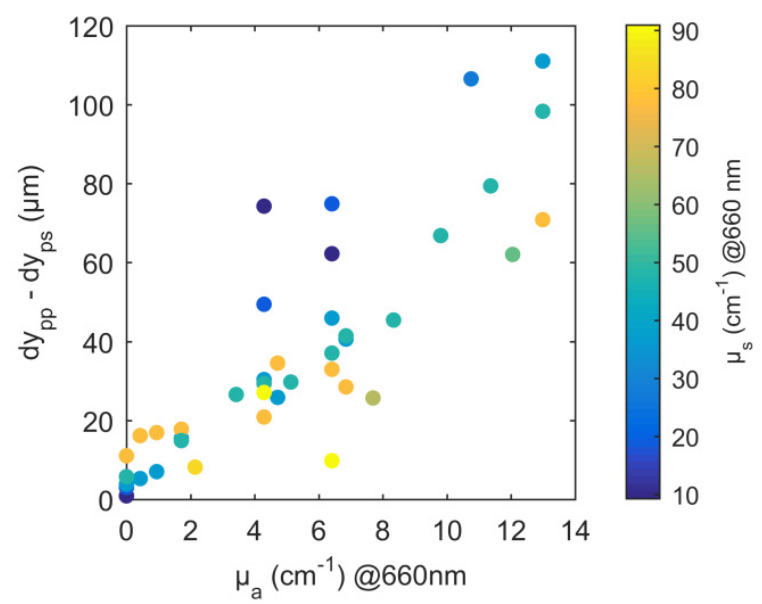

Fig. 11. $d y_{p p}-d y_{p s}$ values versus $\mu_{a}$. The colorbar gives the level of scattering, $\mu_{s}$, for each point.

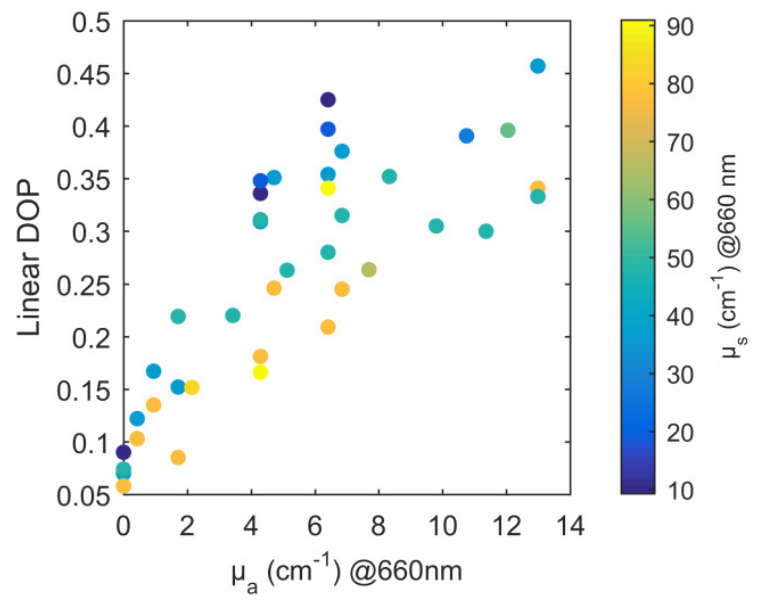

Fig. 12. Linear DOP values versus $\mu_{a}$. The colorbar gives the level of scattering, $\mu_{s}$, for each point.

Again, as shown on Fig. 10, predicted values of $\mu_{a}$ at $660 \mathrm{~nm}$ can be improved by using stepwise-MLR model with two parameters ( $\mathrm{R}^{2}$ goes from 0.740 to $0.918, \mathrm{RMSE}_{\mathrm{c}}$ from 2.1 to $\left.1.2 \mathrm{~cm}^{-1}\right): d y_{p p}-d y_{p s}$ and $C_{p p}$.

From Eq.(18), for a given value of $d y_{p p}-d y_{p s}, C_{p p}$ increases when $\mu_{a}$ increases. The opposite was observed between $C_{p p}$ and $\mu_{s}$ at $405 \mathrm{~nm}$ [Eq. (10)]. Using the same arguments, a high $C_{p p}$ value implies a lower number of independent speckle patterns [Eq.(14)]. Here, when $\mu_{a}$ value is high, light will not propagate deeply inside the sample and there will be fewer independent speckle patterns due to depolarization effects. Hence $C_{p p}$ value increases with $\mu_{a}$ value. Moreover, one can notice that adding $C_{p p}$ value to the $\mu_{a}$-model equation improves predictions regardless of the sample $\mu_{s}$ value.

In the case of a scattering and absorbing medium, it is interesting to notice that $\mu_{a}$ and $\mu_{s}$ coefficients are predicted using parameters derived from the polarization analysis (both $d y_{p p}$ and $d y_{p s}$ are used). According to results found in [31, 40], further investigations using circular polarizations could be considered to improve the calibration models.

\subsection{Prediction ability of regression models}


The prediction ability of all three regression models was evaluated using one quarter of the 41 liquid optical phantoms defined in 2.1.1. The 11 samples used to test our models were chosen in such a way as to be representative among the calibration set. This means that $\mu_{s}$ and $\mu_{a}$ standard deviations in the test samples were close to those used for the calibration: $\sigma\left(\mu_{s, \text { calib set }}\right) \approx \sigma\left(\mu_{s, \text { test set }}\right)$ and $\sigma\left(\mu_{a, \text { calib set }}\right) \approx \sigma\left(\mu_{a, \text { test set }}\right)$.

\subsubsection{Case of scattering media}

Fig. 13 shows $\mu_{s}$ predicted values at $405 \mathrm{~nm}$ for calibration and test datasets using the 2parameter regression model described in Section 3.B [Eq. (10)].

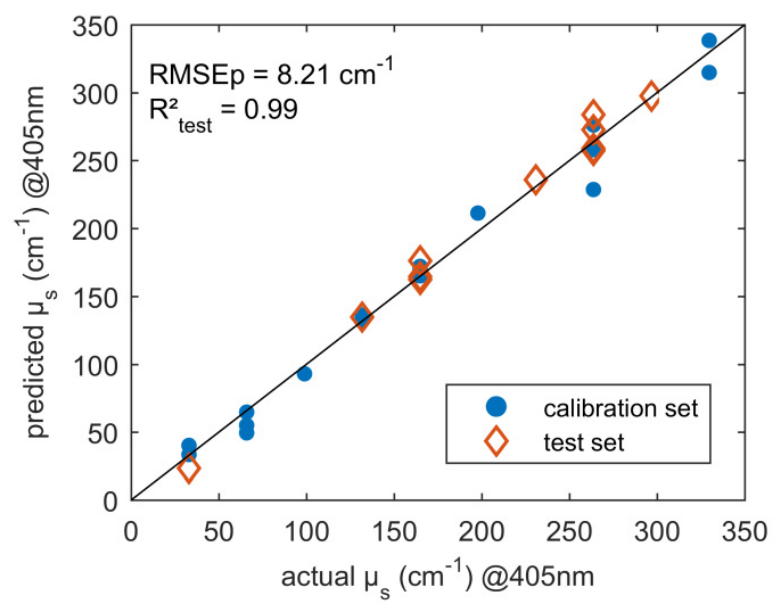

Fig. 13. Stepwise MLR predicted values versus actual values of $\mu_{s}$ at $405 \mathrm{~nm}$ using two parameters, for calibration and test datasets.

First of all, the graph (Fig. 13) confirms that calibration and test samples cover the same $\mu_{s}$ value ranges. In addition, $\mathrm{R}^{2}$ values for prediction and calibration (see Fig. 3.b) are in the same order of magnitude $(\sim 0.99)$. The same holds true for RMSEc and RMSEp values ( $\sim 8.5$ $\mathrm{cm}^{-1}$ ). These results show that the prediction ability of the 2-parameter $\mu_{s}$ model is very efficient in the case of scattering media.

\subsubsection{Case of scattering and absorbing media}

\section{$\mu_{s}$ prediction}

Similarly, Fig. 14 shows $\mu_{s}$ predicted values at $660 \mathrm{~nm}$ for calibration and test datasets using the 2-parameter regression model described in Section 3.2 [Eq.(16)]. 


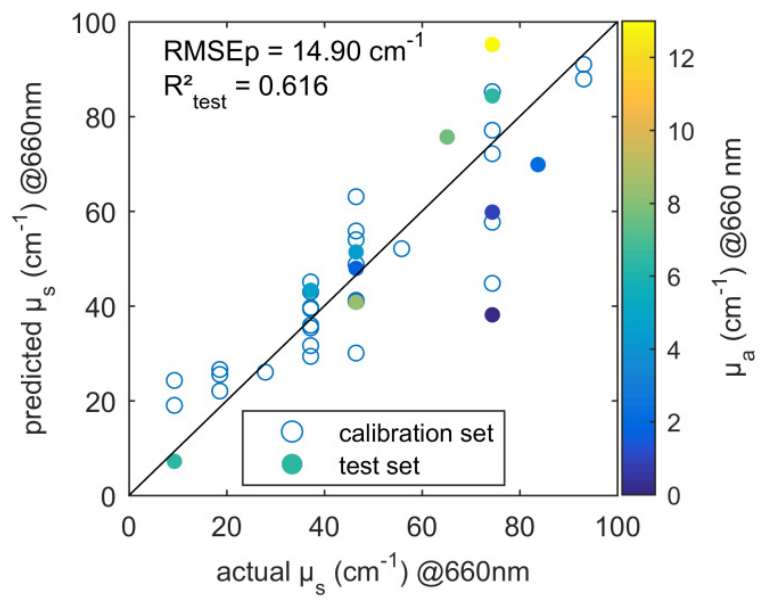

Fig. 14. Stepwise MLR predicted values versus actual values of $\mu_{s}$ at $660 \mathrm{~nm}$ using two parameters, for calibration and test datasets. The colorbar gives the level of absorption, $\mu_{a}$, for each test point.

In this case, the prediction ability of $\mu_{s}$ model is not as accurate as previously. The $\mathrm{R}^{2}$ value obtained for prediction is lower than the one obtained for calibration (0.81 see Fig. 7.b), whereas the RMSEp is higher than the RMSEc $\left(10.1 \mathrm{~cm}^{-1}\right)$. On Fig. 14, the test points are colored according to their absorption level. This clearly shows that the model prediction ability is negatively impacted by samples possessing the same $\mu_{s}=75 \mathrm{~cm}^{-1}$ but with large variations of $\mu_{a}$ values. When scattering and absorbing coefficients vary in these proportions, the proposed method to predict $\mu_{s}$ at $660 \mathrm{~nm}$ might not be suitable, especially for high levels of scattering.

The prediction model of $\mu_{s}$ at $660 \mathrm{~nm}$ may also be improved by adding parameters derived from $405 \mathrm{~nm}$ measurements or by using nonlinear regression models. However, physics interpretations would be more difficult to make than those made with stepwise-MLR results.

\section{$\mu_{a}$ prediction}

Fig. 15 shows $\mu_{a}$ predicted values at $660 \mathrm{~nm}$ for calibration and test datasets using the 2parameter regression model described in Section 3.2 [Eq. (18)].

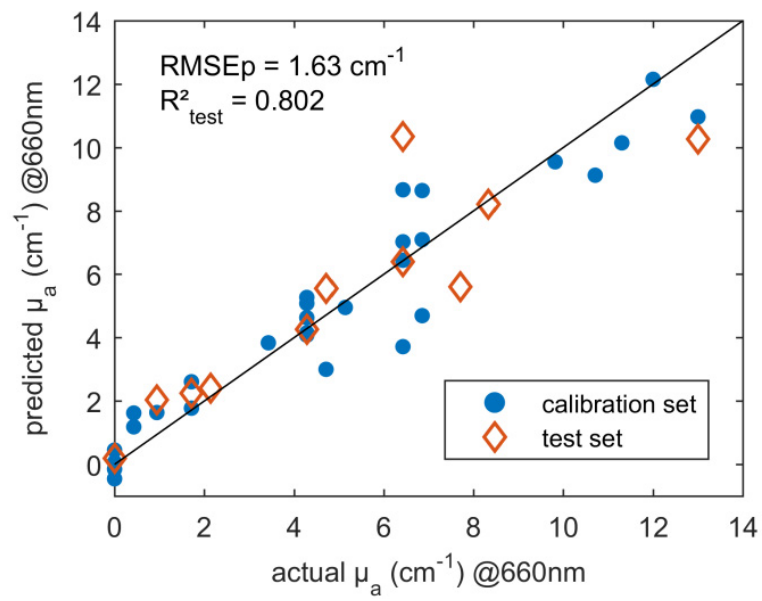

Fig. 15. Stepwise MLR predicted values versus actual values of $\mu_{a}$ at $660 \mathrm{~nm}$ using two parameters, for calibration and test datasets. 
The prediction ability of the 2-parameter $\mu_{a}$ model is slightly lower when compared to results obtained during calibration (see Fig. 10.b): the $\mathrm{R}^{2}$ value is lower whereas the RMSEp is somewhat higher than the RMSEc. However, the results are very encouraging. Indeed, the proposed method predicts $\mu_{a}$ in scattering and absorbing media with satisfying accuracy.

\section{Conclusion}

This work investigated the usefulness of combining polarization, backscattered speckle and chemometric approaches, to predict optical absorption and scattering coefficients $\left(\mu_{a}\right.$ and $\left.\mu_{s}\right)$ of liquid samples at $405 \mathrm{~nm}$ and $660 \mathrm{~nm}$.

At $405 \mathrm{~nm}$, the studied samples only exhibited scattering behavior whereas at $660 \mathrm{~nm}$, they had both scattering and absorbing properties. The prediction ability of the regression models were then evaluated in these two cases.

Linear models were built with stepwise multiple linear regressions, to predict $\mu_{a}$ or $\mu_{s}$ using two variables computed from the polarized speckle patterns. In the case of scattering media (samples measured at $405 \mathrm{~nm}$ ), $\mu_{s}$ was predicted with a very good accuracy with a model using $d y_{p p}$ and $C_{p p}\left(\mathrm{R}^{2}>0.9\right)$. However, in the case of scattering and absorbing media (samples measured at $660 \mathrm{~nm}$ ), $\mu_{s}$ model was built with $d y_{p p}$ and $d y_{p s}$ and yielded less accurate prediction results $\left(\mathrm{R}^{2}=0.62\right)$. Besides, $\mu_{a}$ was predicted with a model using $d y_{p p}$ $d y_{p s}$ and $C_{p p}$, giving satisfying results $\left(\mathrm{R}^{2}=0.8\right)$.

The proposed method, by combining polarized speckle parameters and stepwise-MLR yields fairly good results in predicting bulk optical properties $\left(\mu_{s}\right.$ or $\left.\mu_{a}\right)$. However, it seems more difficult to predict $\mu_{s}$ in the case of absorbing media. The prediction model for $\mu_{s}$ at 660 $\mathrm{nm}$ could be improved by (1) adding parameters from $405 \mathrm{~nm}$ measurements to build stepwise-MLR models, (2) taking into account all parameters and use Partial Least Square Regressions, (3) including non-linear terms to build the models or (4) using non-linear methods such as Neural Network or Support Vector Machine.

Further investigation should be carried out on complex biological samples (such as plant leaves, soil, waste...) in order to assess the potential of this method on biological complex media. Indeed, this cost-effective and non-invasive method could lead to the development of new sensors.

\section{Funding}

French National Research Agency (ANR-16-CE04-0010)

\section{Acknowledgments}

This work was conducted within the OPTIPAG Project supported by the grant ANR-16CE04-0010 from the the French National Research Agency.

\section{Disclosures}

The authors declare that there are no conflicts of interest related to this article.

\section{References}

1. V. V. Tuchin, Tissue Optics: Light Scattering Methods and Instruments for Medical Diagnosis, 2nd ed. (SPIE Press, 2007), p. 840

2. A. N. Bashkatov, E. A. Genina, V. I. Kochubey, and V. V. Tuchin, "Optical properties of human skin, subcutaneous and mucous tissues in the wavelength range from 400 to $2000 \mathrm{~nm}$ ”, J. Phys. D Appl. Phys. 38(15), 2543-2555 (2005).

3. W. Saeys, M. A. Velazco-Roa, S. N. Thennadil, H. Ramon, and B. M. Nicolaï, "Optical properties of apple skin and flesh in the wavelength range from 350 to $2200 \mathrm{~nm}$," Appl. Opt. 47(7), 908-919 (2008). 
4. S Bellini, R Bendoula, E Latrille, JM Roger, "Potential of a spectroscopic measurement method using addingdoubling to retrieve the bulk optical properties of dense microalgal media", Applied spectroscopy 68 (10), 1154-1167 (2014)

5. Y.-C. Chen and S. N. Thennadil, "Insights into information contained in multiplicative scatter correction parameters and the potential for estimating particle size from these parameters," Anal. Chim. Acta 746, 37-46 (2012).

6. M. Rey-Bayle, R. Bendoula, S. Henrot, K. Lamiri, F. Baco-Antoniali, N. Caillol, A. Gobrecht, J.M Roger, "Potential of vis-NIR spectroscopy to monitor the silica precipitation reaction", Analytical and bioanalytical chemistry 409 (3), 785-796 (2017).

7. T. Farrell, M. Patterson, B. Wilson. “A Diffusion Theory Model of Spatially Resolved, Steady-State Diffuse Reflectance for the Noninvasive Determination of Tissue Optical Properties in vivo", Med. Phys. 19(4), 879888 (1992).

8. F. Chauchard, J.M. Roger, V. Bellon-Maurel, C. Abrahamsson, S. Andersson-Engels, S. Svanberg. "MADSTRESS: A Linear Approach for Evaluating Scattering and Absorption Coefficients of Samples Measured Using Time-Resolved Spectroscopy in Reflection'. Appl. Spectrosc. 59(10): 1229-1235 (2005).

9. C. Abrahamsson, J. Johansson, S. Andersson-Engels, S. Svanberg, S. Folestad. "Time-Resolved NIR Spectroscopy for Quantitative Analysis of Intact Pharmaceutical Tablets". Anal. Chem. 77(4): 1055-1059 (2005).

10. S. Torrance, Z. Sun, E. Sevick-Muraca. "Impact of Excipient Particle Size on Measurement of Active Pharmaceutical Ingredient Absorbance in Mixtures Using Frequency Domain Photon Migration'. J.Pharm. Sci. 93(7): 1879-1889 (2004).

11. R. Steponavicius, S.N. Thennadil. "Extraction of Chemical Information of Suspensions Using Radiative Transfer Theory to Remove Multiple Scattering Effects: Application to a Model Multicomponent System'” Anal. Chem. 83(6): 1931-1937 (2011).

12. R. Steponavicius, S.N. Thennadil. "Extraction of Chemical Information of Suspensions Using Radiative Transfer Theory to Remove Multiple Scattering Effects: Application to a Model Two-Component System". Anal. Chem. 81(18): 7713-7723 (2009).

13. A. Ghabbach, M. Zerrad, G. Soriano, and C. Amra, "Accurate metrology of polarization curves measured at the speckle size of visible light scattering," Opt Express 22, 14594-14609 (2014).

14. A. Ghabbach, M. Zerrad, G. Soriano, S. Liukaityte, and C. Amra, "Depolarization and enpolarization DOP histograms measured for surface and bulk speckle patterns," Opt Express 22, 21427-21440 (2014).

15. J. Swartling, J. Dam, S. Andersson-Engels. "Comparison of Spatially and Temporally Resolved DiffuseReflectance Measurement Systems for Determination of Biomedical Optical Properties", Appl. Opt. 42(22): 4612-4620 (2003).

16. J. W. Goodman, "Statistical properties of laser speckle patterns, " in Laser Speckle and Related Phenomena, J. C. Dainty, Ed., Vol. 9 in series Topics in Applied Physics, pp. 9 - 75, Springer, Berlin, Heidelberg (1975).

17. K. Ishii, T. Iwai, S.Wada, and M. Miyakoshi, "Simultaneous viscometry and particle sizing on the basis of dynamic light scattering," Proc. SPIE 4263, 112-121 (2001).

18. D.A. Boas and A. G. Yodh, "Spatially varying dynamical properties of turbid media probed with diffusing temporal light correlation,” J. Opt. Soc. Am. A 14, 192-215 (1997).

19. J.D. Briers, G. Richards and X.W. He, "Capillary blood flow monitoring using laser speckle contrast analysis (LASCA)," J. Biomed. Opt. 4, 164-175 (1999).

20. D.A. Zimnyakov, J.D. Briers, V.V. Tuchin, "Speckle technologies for monitoring and imaging of tissues and tissuelike phantoms," Chap.18 in Handbook of biomedical diagnostics, Valery V. Tuchin, Ed. (SPIE press, Bellingham 2002).

21. A. Zdunek, A. Adamiak, P. M. Pieczywek, A. Kurenda, "The biospeckle method for the investigation of agricultural crops: A review", Optics and Lasers in Engineering 52, 276-285 (2014).

22. J. Sorrentini, M. Zerrad, G. Soriano, and C. Amra, "Enpolarization of light by scattering media," Opt Express 19, 21313-21320 (2011).

23. M. Zerrad, J. Sorrentini, G. Soriano, and C. Amra, "Gradual loss of polarization in light scattered from rough surfaces: Electromagnetic prediction," Opt Express 18, 15832-15843 (2010).

24. M. Zerrad, H. Tortel, G. Soriano, A. Ghabbach, and C. Amra, "Spatial depolarization of light from the bulks: electromagnetic prediction," Opt. Express 23, 8246-8260 (2015).

25. G. Anna, F. Goudail, and D. Dolfi, "Optimal configurations for active polarimetric imaging systems in the presence of different sources of Poisson shot noise," Opt. Commun. 291, 116-123 (2013).

26. J. Dupont, X. Orlik, A. Ghabbach, M. Zerrad, G. Soriano, and C. Amra, "Polarization analysis of speckle field below its transverse correlation width : application to surface and bulk scattering," Opt. Express 22, 24133-24141 (2014).

27. J. Dupont, X. Orlik, R. Ceolato, and T. Dartigalongue, "Spectralon spatial depolarization: towards an intrinsic characterization using a novel phase shift distribution analysis," Opt. Express 25, 9544 (2017).

28. J. Broky and A. Dogariu, "Correlations of polarization in random electro-magnetic fields," Opt. Express 19, 15711-15719 (2011).

29. A. H. Hielscher, J. R. Mourant, and I. J. Bigio, “Influence of particle size and concentration on the diffuse backscattering of polarized light from tissue phantoms and biological cell suspensions, " Appl. Opt. 36, 125 135 (1997). 
30. Z. Hajjarian and S. K. Nadkarni, "Estimation of particle size variations for laser speckle rheology of materials, "Opt. Lett. 40, $764-767$ 2015).

31. C. Abou Nader, R Nassif, F. Pellen, B Le Jeune, G. Le Brun, M. Abboud, "Influence of size, proportion, and absorption coefficient of spherical scatterers on the degree of light polarization and the grain size of speckle pattern", Applied Optics 54, 10369 - 10376 (2015).

32. K. Kahaksari, S. J. Kirkpatrick, "Combined effects of scattering and absorption on laser speckle contrast imaging", Journal of biomedical optics 21, 1-8 (2016).

33. B. Aernouts, E. Zamora-Rojas, R. Van Beers, R. Watté, L. Wang, M. Tsuta, J. Lammertyn, W. Saeys, "Supercontinuum laser based optical characterization of Intralipid ${ }^{\circledR}$ phantoms in the $500-2250 \mathrm{~nm}$ range," Opt. Express 21, 32450-32467 (2013).

34. R. Michels, F. Foschum, and A. Kienle, "Optical properties of fat emulsions", Opt. Express 16(8), 5907-5925 (2008).

35. B. Cletus, R. Künnemeyer, P. Martinsen, and V. A. McGlone, "Temperature-dependent optical properties of Intralipid measured with frequency-domain photon-migration spectroscopy," J. Biomed. Opt. 15(1), 017003 (2010).

36. P. D. Ninni, F. Martelli, and G. Zaccanti, "Intralipid: towards a diffusive reference standard for optical tissue phantoms," Phys. Med. Biol. 56(2), N21-N28 (2011).

37. P. I. Rowe, R. Künnemeyer, A. McGlone, S. Talele, P. Martinsen, and R. Oliver, "Thermal stability of intralipid optical phantoms," Appl. Spectrosc. 67(8), 993-996 (2013).

38. M. N. Usacheva, M. C. Teichert, and M. A. Biel, "The role of the methylene blue and toluidine blue monomers and dimers in the photoinactivation of bacteria," J. Photochem. Photobiol. B 71(1-3), 87-98 (2003).

39. S. Prahl, Oregon Medical Laser Center, "Methylen Blue Spectra", https://omlc.org/spectra/mb/index.html

40. Y. Piederriere, F. Boulvert, J. Cariou, B. Le Jeune, Y.Guern, G. Le Brun, "Backscattered speckle size as a function of polarization: influence of particle-size and -concentration" Opt. Express 13, 5030 (2005)

41. R Bendoula, A Gobrecht, B Moulin, JM Roger, V Bellon-Maurel, "Improvement of the chemical content prediction of a model powder system by reducing multiple scattering using polarized light spectroscopy", Applied spectroscopy 69 (1), 95-102 (2015).

42. Y. Piederrière, J. Cariou, Y. Guern, B. Le Jeune, G. Le Brun, J. Lotrian, "Scattering through fluids: speckle size measurement and Monte Carlo simulations close to and into the multiple scattering," Opt. Express 12,176-188 (2004).

43. Q.B. Li, F.P. Chiang, “Three-dimensional dimension of laser speckle”, Applied Optics 31 (29), 6287-6291 (1992).

44. M. Boffety, M Allain, A Sentenac, M Massonneau, R Carminati, "Analysis of the depth resolution limit of luminescence diffuse optical imaging.", Optics Letters 3 (20):2290-2292 (2008).

45. J. Crassous, M.Erpelding, A Amon, "Diffusive wave spectroscopy applied to the spatially resolved deformation of a solid", Phys Rev E Stat Nonlin Soft Matter Phys 78(4), 046104 (2008).

46. S. P. Morgan and M. E. Ridgway, "Polarization properties of light backscattered from a two layer scattering medium," Opt. Express 7, 395-402 (2000).

47. A. Gobrecht, .R Bendoula, J-M Roger, V. Bellon-Maurel, "Combining linear polarization spectroscopy and the Representative Layer Theory to measure the Beer-Lambert law absorbance of highly scattering materials", Analytica Chimica Acta 853 486-494 (2015).

48. G. Soriano, M. Zerrad, and C. Amra, "Enpolarization and depolarization of light scattered from chromatic complex media," Opt. Express 22, 12603-12613 (2013).

49. E. Jakeman, and K. D. Ridley. Modeling Fluctuations in Scattered Waves. Taylor and Francis Group, ed., 2006.

50. H. Martens and T. Naes, "Multivariate calibration," in Chemometrics (Springer, 1984), pp. 147-156. 